\title{
Clinicopathological and prognostic value of SIRT6 in patients with solid tumors: a meta-analysis and TCGA data review
}

\author{
Xiaojing Wu ${ }^{1,2+}$, Shuyuan Wang ${ }^{2 \dagger}$, Xuanzhu Zhao ${ }^{1,3 \dagger}$, Sizhen Lai ${ }^{1,3 \dagger}$, Zhen Yuan ${ }^{2}$, Yixiang Zhan ${ }^{1,2}, K_{\text {Kemin Ni }}{ }^{1,2}$,

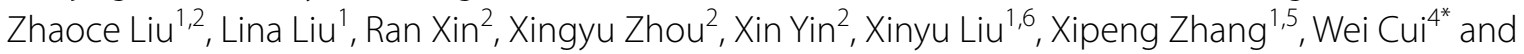 \\ Chunze Zhang ${ }^{1,5^{*}}$ (1)
}

\begin{abstract}
Purposes: In addition to its role in cellular progression and cancer, SIRT6, a member of nicotinamide adenine dinucleotide $\left(\mathrm{NAD}^{+}\right)$-dependent class III deacylase sirtuin family, serves a variety of roles in the body's immune system. In this study, we sought to determine the relationship between the expression of SIRT6 and the clinicopathological outcomes of patients with solid tumours by conducting a meta-analysis of the available data.

Methods: The databases PubMed and ISI Web of Science were searched for relevant literature, and the results were presented here. Using Stata16.0, a meta-analysis was conducted to determine the impact of SIRT6 on clinicopathological characteristics and prognosis in malignancy patients. The results were published in the journal Cancer Research. The dataset from the Cancer Genome Atlas (TCGA) was used to investigate the prognostic significance of SIRT6 in various types of tumors.

Results: The inclusion and exclusion criteria were met by 15 studies. In patients with solid tumours, reduced SIRT6 expression was found to be related with improved overall survival (OS) $(\mathrm{HR}=0.66,95 \% \mathrm{Cl}=0.45-0.97, \mathrm{P}<0.001)$ as well as improved disease-free survival (DFS) ( $H R=0.48,95 \% \mathrm{Cl}=0.26-0.91, \mathrm{P}<0.001)$. Low SIRT6 expression was found to be associated with a better OS in breast cancer ( $\mathrm{HR}=0.49,95 \% \mathrm{Cl}=0.27-0.89, \mathrm{P}=0.179)$, but was found to be associated with a worse OS in gastrointestinal cancer (gastric cancer and colon cancer) $(\mathrm{HR}=1.83,95 \% \mathrm{Cl}=1.20-2.79$, $P=0.939)$ after subgroup analysis. In terms of clinicopathological characteristics, SIRT6 expression was found to be linked with distant metastasis $(\mathrm{OR}=2.98,95 \% \mathrm{Cl}=1.59-5.57, \mathrm{P}=0.694)$. When the data from the TCGA dataset was compared to normal tissue, it was discovered that SIRT6 expression was significantly different in 11 different types of cancers. Meanwhile, reduced SIRT6 expression was shown to be associated with improved OS $(P<0.05)$, which was consistent with the findings of the meta-analysis. Aside from that, the expression of SIRT6 was found to be associated with both gender and clinical stage.
\end{abstract}

\footnotetext{
*Correspondence: weicui@nankai.edu.cn; chunze.zhang@nankai.edu.cn

${ }^{\dagger}$ Xiaojing Wu, Shuyuan Wang, Xuanzhu Zhao and Sizhen Lai contributed equally to this work

${ }^{1}$ Department of Colorectal Surgery, Tianjin Union Medical Center, Tianjin 300121, China

${ }^{4}$ School of Mathematical Sciences and LPMC, Nankai University, Tianjin 300071, China

Full list of author information is available at the end of the article
}

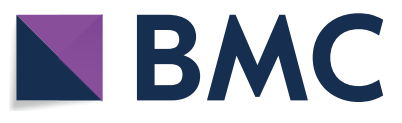

(c) The Author(s) 2022. Open Access This article is licensed under a Creative Commons Attribution 4.0 International License, which permits use, sharing, adaptation, distribution and reproduction in any medium or format, as long as you give appropriate credit to the original author(s) and the source, provide a link to the Creative Commons licence, and indicate if changes were made. The images or other third party material in this article are included in the article's Creative Commons licence, unless indicated otherwise in a credit line to the material. If material is not included in the article's Creative Commons licence and your intended use is not permitted by statutory regulation or exceeds the permitted use, you will need to obtain permission directly from the copyright holder. To view a copy of this licence, visit http://creativecommons.org/licenses/by/4.0/. The Creative Commons Public Domain Dedication waiver (http://creativeco mmons.org/publicdomain/zero/1.0/) applies to the data made available in this article, unless otherwise stated in a credit line to the data. 
Conclusion: The overall data of the present meta-analysis indicated that low expression of SIRT6 may predict a favorable survival for patients with solid tumors.

Keywords: SIRT6, Solid tumors, Prognosis, Meta-analysis

\section{Introduction}

Sirtuins, a conserved protein family, are identified to have the deacylase and/or mono-ADP-ribosyltransferase activities [1]. Sirtuins have been implicated in a variety of physiological processes, including transcription, DNA repair, tumorigenesis, metabolism, stress responses, apoptosis, fat mobilization, and aging [2]. Among seven identified members (SIRT1-7) of these histone deacetylases, sirtuin 6 (SIRT6) has been shown to be involved in cellular pathways and to play a critical role in regulating ageing and sugar metabolism, both of which are associated with the occurrence and development of tumours and are thus significantly associated with cancer patient prognosis [3, 4]. Against this background, SIRT6 is considered to be a regulator in the progression of cancer and thus affect the survival rate of cancer patients.

SIRT6 expression levels vary significantly between tumour types. Researches reveal that SIRT6 is overexpressed in osteosarcoma [5], papillary thyroid cancer (PTC) [6], prostate cancer [7], conversely reduced in renal cell carcinoma (RCC) [8], pancreatic ductal adenocarcinoma (PDAC) [9], colon cancer (CRC) [10], non-small cell lung cancer (NSCLC) [11]. On the other hand, SIRT6 is thought to play a role in or suppress the progression of several types of cancer. For example, immunohistochemistry revealed that SIRT6 expression was considerably lower in tumour tissues than in normal tissues in RCC patients, implying that SIRT6 worked as a tumour suppressor [8]. Loss of SIRT6 expression in human PDAC defined a subset of patients with a worse prognosis [9]. Moreover, Lower SIRT6 levels were demonstrated in colon cancer and were associated with shorter survival than those of patients with higher SIRT6 expression [10].

In comparison, patients with osteosarcoma who express a high level of SIRT6 exhibit malignant clinical features and have a worse survival rate, with in vitro experiments indicating that SIRT6 overexpression aided MG63 cell motility and invasion [5]. In NSCLC cell lines and tumor tissues, SIRT6 is proved to be upregulated, and statistical analyses showed that high SIRT6-expressing NSCLC patients had a lower cumulative survival rate as compared with low SIRT6expression patients [11]. SIRT6 upregulation was associated with poor recurrence-free survival (RFS) in PTC patients, given that patients with the higher expression of SIRT6 had the worse RFS and those who possessed lower expression of the gene had the better RFS [6]. Besides, high SIRT6 expression was associated with poor OS of gastric cancer [12].

As a result, while the molecular pathways behind SIRT6 have been explored, the relationship between SIRT6 expression and the prognosis of patients with solid malignancies remains contentious. The goal of this study is to further elucidate the role of SIRT6 in mammalian solid tumours by meta-analysis and the TCGA dataset, which may aid in the detection and treatment of certain cancers.

\section{Materials and methods Study research}

We conducted an electronic literature search of all publications in the PubMed and ISI Web of Science databases to determine whether there is a correlation between SIRT6 expression and survival in solid tumours. The research was terminated on August 15, 2021, with no lower date limit. Sirtuin 6, SIRT6, cancer, tumour, prognosis, prognostic, and survival were all included in the search terms in all possible combinations. Searches were limited to human studies and those published in English.

\section{Inclusion and exclusion criteria}

The included studies had to meet the following criteria: (1) to be published in its entirety in the English language; (2) to make a pathological diagnosis of cancer; (3) to describe the pathological diagnosis of various tumour types or clinicopathological features; (4) to measure SIRT6 expression in patients with any type of tumour via immunohistochemistry; (5) to describe associations between SIRT6 expression and OS and DFS; and (6) to report or calculate HRs and 95\% CIs based on the information in the pamphlet.

The following criteria were used to exclude studies from this meta-analysis: (1) Reviews, letters, comments, repetitive research, case reports, or personal communications; (2) non-English language articles; (3) articles that overlap or contain duplicate data; (4) articles that contain only animal experiments; and (5) studies that do not include survival curves or data on survival.

\section{Data extraction}

Two investigators (Shuyuan Wang and Zhen Yuan) extracted all data independently based on the inclusion 
and exclusion criteria, and all items were finally agreed upon. The following characteristics were extracted for each eligible study: the first author's name, the publication year, the region, the type of cancer, the number of patients, the duration of follow-up, the detection methods, the survival data (including OS and DFS), and clinicopathological parameters such as gender, tumour differentiation, $\mathrm{T}$ status, lymph node metastasis, distant metastasis, and TNM stage.

\section{Statistical analysis}

Stata 16.0 (Stata Corporation, College Station, TX, USA) was used to conduct this meta-analysis. The association between SIRT6 expression and survival outcome was evaluated using pooled HR estimates with $95 \%$ CIs, while the association with clinical parameters such as gender, tumour differentiation, lymph node metastasis, distant metastasis, and clinical stage was evaluated using OR estimates with 95\% CIs. Further, the Cochrane's Q test and $\mathrm{I}^{2}$ statistical test were used to analyze the heterogeneity between studies, the fixed effects model (Mantel-Haenszel method) was conducted when heterogeneity was negligible $\left(\mathrm{I}^{2}<50 \%\right)$, and a random effects model (DerSimonian-Laird method) was used when heterogeneity was significant $\left(\mathrm{I}^{2}>50 \%\right)$. Begg's funnel plot was conducted to identify publication bias. All the $P$ values were used for a two-sided test with significance at $\mathrm{P}<0.05$. The $\mathrm{HRs}$ and $95 \% \mathrm{CIs}$ were extracted from articles that only reported Kaplan-Meier curves using Software Engauge Digitizer (version 10.8). Tierney et al. provided the method and EXCEL programmed for calculating the data [13].

\section{Extraction and analysis of TCGA dataset}

The Cancer Genome Atlas (TCGA) dataset (https://www. cancer.gov/about-nci/organization/ccg/research/struc tural-genomics/tcga) and UCSC Xena project (https:// xena.ucsc.edu/) were used to extract the data in malignant tumors and normal tissues, including the expression of SIRT6, survival data and clinicopathological parameters. The differential expression of SIRT6 between tumours and paired normal tissues was analyzed using Wilcoxon's signed-rank test. The OS and DFS curves were generated using the Kaplan-Meier method and the logrank test, respectively, using data from the TCGA dataset. To analyse the prognostic effects of SIRT6 in various tumours, the forest plot was mapped using univariate Cox regression. The correlation between SIRT6 expression and clinicopathological parameters were determined using a one-way ANOVA. All data were analyzed and plots were created using the $\mathrm{R}$ programming language (version 3.6.1).

\section{Results}

Literature search and study characteristics

A total of 1103 publications were discovered in the PubMed and ISI Web of Science databases. Figure 1 depicts a detailed study selection. 1088 of those were excluded due to duplication of research, lack of complete texts, lack of information about survival, detection method, or studies irrelevant to the current analysis. Finally, 15 publications were included in this study, totaling 1577 patients. Table 1 summaries the major characteristics of the 15 eligible studies.

\section{Decreased SIRT6 expression and overall survival}

The association between SIRT6 expression and the prognosis for OS was estimated; the pooled HR and 95\% CIs are shown in Fig. 2A. The findings demonstrated that low SIRT6 expression was associated with a longer overall survival in patients with solid cancer $(\mathrm{HR}=0.66,95 \% \mathrm{CI}=0.45-0.97, \mathrm{P}<0.001)$. As shown in Fig. 2B, we conducted a subgroup analysis by cancer type, which revealed that low SIRT6 expression was significantly associated with better overall survival in breast cancer $(\mathrm{HR}=0.49,95 \% \mathrm{CI}=0.27-0.89$, $\mathrm{P}=0.179$ ) and other system cancer (OS and $\mathrm{OC}$ ) $(\mathrm{HR}=0.30,95 \% \mathrm{CI}=0.10-0.91, \mathrm{P}=0.069)$, but not in non-small cell lung cancer, hepatobiliary and pancreatic cancer. However, Furthermore, decreased SIRT6 expression was found to be associated with a shorter overall survival (OS) in gastrointestinal tumours (GC and $\mathrm{CRC})(\mathrm{HR}=1.83,95 \% \mathrm{CI}=1.20-2.79, \mathrm{P}=0.939)$.

\section{Decreased SIRT6 expression and disease-free survival}

The association between SIRT6 expression and prognosis for DFS has been estimated; the pooled HRs and 95\% CIs are shown in Fig. 3. In a multivariate analysis of patients with solid tumours, a significant correlation between attenuated SIRT6 expression and DFS $(\mathrm{HR}=0.48,95 \% \mathrm{CI}=0.26-0.91)$ was observed in the random effects model with a significant heterogeneity $\left(\mathrm{I}^{2}=83.8 \%, \mathrm{P}<0.001\right)$.

\section{Correlations between Low SIRT6 Expression and Clinicopathological parameters}

12 eligible articles were used to collect the clinical and pathological parameters, owing to 3 articles [9, 23, 24] did not provide related information, the specific parameters were presented in Additional file 4: Table S1. As shown in Table 2, correlations between attenuatedexpressed SIRT6 and clinicopathological characteristics of patients with solid tumours were discovered using pooled results of the correlations. Reduced SIRT6 expression was found to be associated with distant 


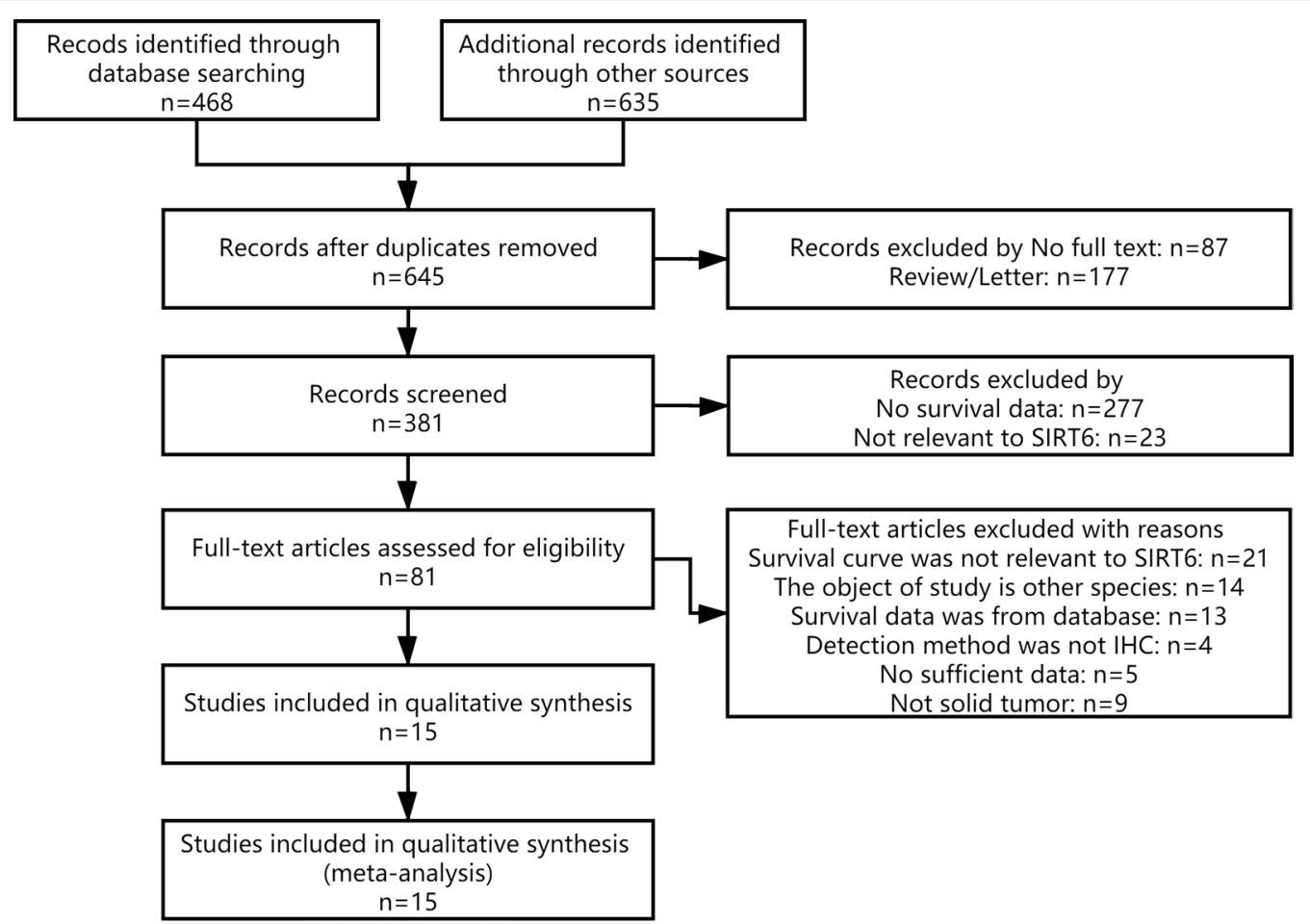

Fig. 1 Flow diagram of the selection of eligible studies

metastasis $(\mathrm{OR}=2.98,95 \% \mathrm{CI}=1.59-5.57, \mathrm{P}=0.694)$. No significant correlations between low expressed SIRT6 and gender $(\mathrm{OR}=0.92,95 \% \mathrm{CI}=0.63-1.33$, $\mathrm{P}=0.226)$, tumor differentiation $(\mathrm{OR}=1.35,95 \%$ $\mathrm{CI}=0.68-2.67, \mathrm{P}=0.000), \mathrm{T}$ status $(\mathrm{OR}=1.24,95 \%$ $\mathrm{CI}=0.70-2.18, \quad \mathrm{P}=0.018)$, lymph node metastasis $(\mathrm{OR}=0.86,95 \% \mathrm{CI}=0.55-1.36, \mathrm{P}=0.010)$ and $\mathrm{TNM}$ stage $(\mathrm{OR}=0.69,95 \% \mathrm{CI}=0.35-1.34, \mathrm{P}=0.001)$ were observed when the P-value was controlled to be $<0.05$ (Additional file 1: Fig. S1).

\section{Assessment of heterogeneity and sensitivity analysis}

Because of the high heterogeneity of the meta-analysis with OS, subgroup analyses were performed according to the SIRT6 location (nucleus or cytoplasm), patients' country (China or other), case number ( $\geq 100$ or not), NOS score $(>7$ or not), and publication year $(\geq 2018$ or $\leq 2017$ ), among other variables. Low expression of SIRT6 in the cytoplasm was associated with improved OS; however, the expression of SIRT6 in the nucleus or in a location that was not reported was not associated with OS. Apart from that, Table 3 shows the pooled HRs and heterogeneities according to all of these variables. All of these subgroup analyses revealed that the I2 value was not significantly lower when the $P$ value was less than 0.05 in any of the groups. Therefore, the subgroup analysis failed to identify the source of extreme heterogeneity (Additional file 2: Fig. S2, Additional file 3: Fig. S3).

Additional to this, a sensitivity analysis was carried out by systematically excluding each study from the aggregated survival meta-analyses, in order to assess the influence of each individual study on the pooled HR of OS (Fig. 4). The results revealed that the pooled estimates of the effect of low-expressed SIRT6 on the OS and DFS of patients with solid tumours did not differ significantly when individual studies were excluded, implying that the findings of this meta-analysis were stable.

\section{Publication bias}

The shapes of the funnel plots for OS, DFS and clinicopathological features of patients were almost symmetrical, indicated that there was no statistically significant difference, therefore no significant publication bias (Figs. 5, 6). Thus, in these incorporated papers, it was found that there was no evidence of significant publication bias.

\section{Validation of the results in TCGA dataset}

For further investigation, the OS and DFS data of patients with expression of SIRT6 in different cancers were extracted from the TCGA dataset, including 17 types of tumors, Adrenocortical carcinoma (ACC), Bladder Urothelial Carcinoma (BLCA), Breast invasive 


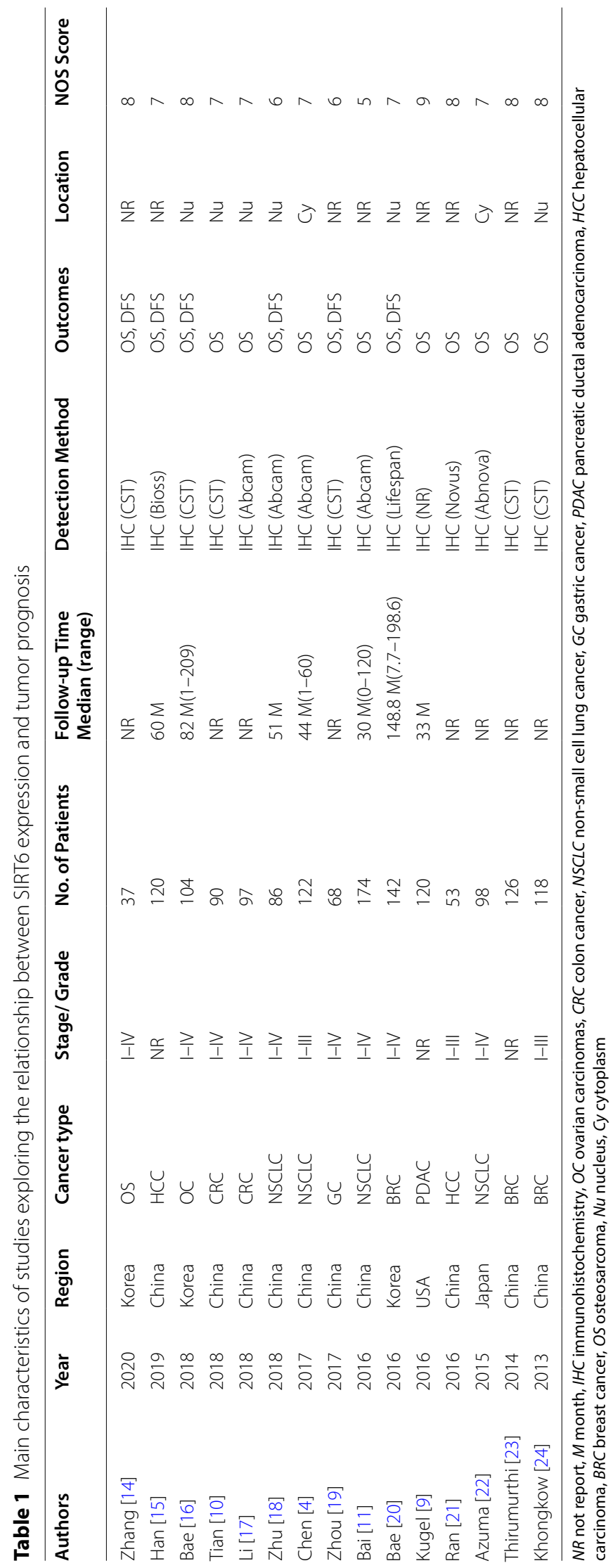


A

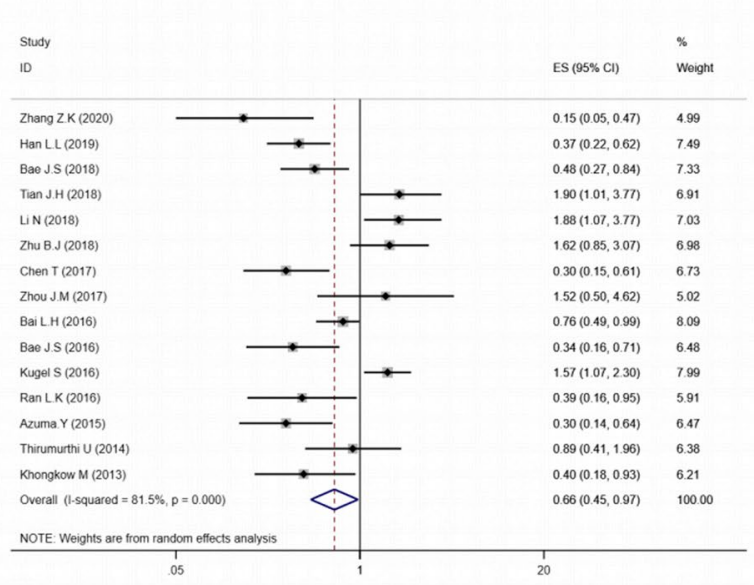

B

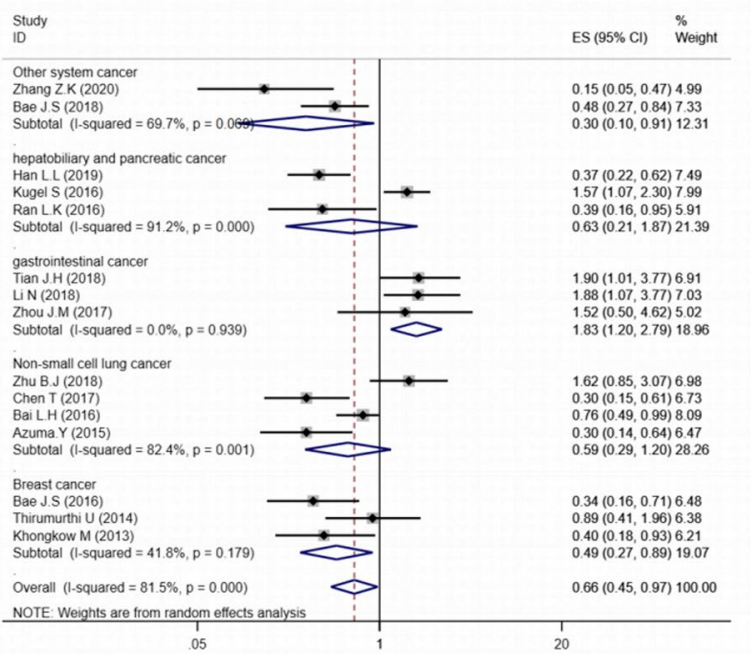

Fig. 2 A Forest plot of the association between SIRT6 expression and OS; B subgroup analysis by tumor type

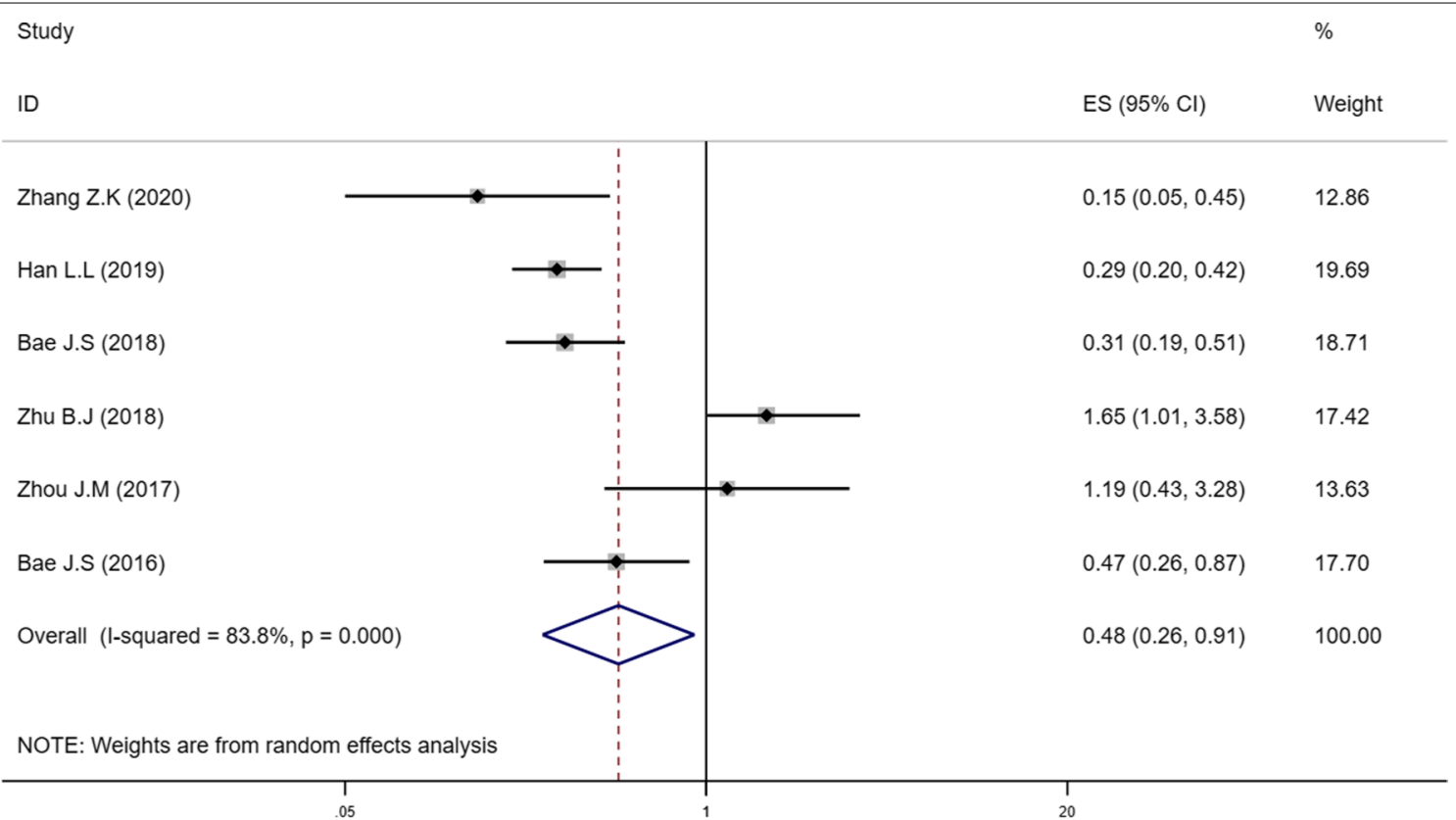

Fig. 3 Forest plot describing the association between low-expressed SIRT6 and DFS

carcinoma (BRCA), Cervical squamous cell carcinoma and endocervical adenocarcinoma (CESC), Cholangiocarcinoma (CHOL), Colon adenocarcinoma (COAD), Esophageal carcinoma (ESCA), Glioblastoma multiforme (GBM), Head and Neck squamous cell carcinoma (HNSC), Kidney Chromophobe (KIRC), Kidney Chromophobe (KICH), Testicular Germ Cell Tumors (TGCT),
Thymoma (THYM), Thyroid carcinoma (THCA), Uterine Corpus Endometrial Carcinoma (UCEC), Uterine Carcinosarcoma (UCS) and Uveal Melanoma (UVM).

As illustrated in Fig. 7, SIRT6 expression was significantly different in 11 different types of malignant tumours compared to normal tissues $(\mathrm{P}<0.05)$. SIRT6 was found to be a high-risk gene in KIRC but a low-risk 
Table 2 Meta-analysis results of the associations of decreased SIRT6 expression with clinicopathological parameters

\begin{tabular}{|c|c|c|c|}
\hline Clinicopathological parameter & Study Number & Overall OR $(95 \% \mathrm{Cl})$ & $I^{2}$ (P-value) \\
\hline Gender (male vs female) & 9 & $0.92(0.63-1.33)$ & $24.5 \%, 0.226$ \\
\hline Tumor differentiation (poor/moderate vs well) & 8 & $1.35(0.68-2.67)$ & $\begin{array}{l}77.8 \% \\
<0.001\end{array}$ \\
\hline T status (T3-4 vs T1-2) & 9 & $1.24(0.70-2.18)$ & $56.7 \%, 0.018$ \\
\hline Lymph node metastasis (yes vs no) & 8 & $0.86(0.55-1.36)$ & $58.7 \%, 0.010$ \\
\hline Distant metastasis (yes vs no) & 3 & $2.98(1.59-5.57)$ & $0.00 \%, 0.694$ \\
\hline TNM stage (III-IV vs I-II) & 7 & $0.69(0.35-1.34)$ & $70.6 \%, 0.001$ \\
\hline
\end{tabular}

If $95 \% \mathrm{Cl}$ value contain 1.0, it means that the $95 \% \mathrm{Cl}$ value intersect the ineffective line and the OR value was no statistical significance

Table 3 Results of subgroup analysis exploring source of heterogeneity with OS and DFS

\begin{tabular}{|c|c|c|c|c|}
\hline \multirow[t]{2}{*}{ Subgroups } & \multicolumn{2}{|l|}{ OS } & \multicolumn{2}{|l|}{ DFS } \\
\hline & $\mathrm{HR}(95 \% \mathrm{Cl})$ & $\mathrm{I}^{2}$ (P-value) & $\mathrm{HR}(95 \% \mathrm{Cl})$ & $\mathrm{I}^{2}$ (P-value) \\
\hline \multicolumn{5}{|l|}{ Location } \\
\hline $\mathrm{Nu}$ & $0.86(0.45-1.66)$ & $82.5 \%(P<0.001)$ & $0.62(0.26-0.91)$ & $88.2 \%(P<0.001)$ \\
\hline Cy & $0.30(0.18-0.50)$ & $0.0 \%(P=1.000)$ & - & - \\
\hline NR & $0.65(0.38-1.13)$ & $81.8 \%(P<0.001)$ & $0.37(0.14-0.97)$ & $76.5 \%(P=0.014)$ \\
\hline \multicolumn{5}{|l|}{ Region } \\
\hline China & $0.79(0.51-1.23)$ & $78.0 \%(P<0.001)$ & $0.80(0.22-2.88)$ & $91.9 \%(P<0.001)$ \\
\hline Other & $0.44(0.19-1.03)$ & $88.4 \%(P<0.001)$ & $0.32(0.19-0.54)$ & $41.8 \%(P=0.180)$ \\
\hline \multicolumn{5}{|c|}{ Patients number } \\
\hline$\geq 100$ & $0.56(0.36-0.88)$ & $80.7 \%(P<0.001)$ & $0.48(0.26-0.91)$ & $0.0 \%(P=0.384)$ \\
\hline$<100$ & $0.80(0.39-1.65)$ & $82.9 \%(P<0.001)$ & $0.70(0.18-2.78)$ & $85.7 \%(P=0.001)$ \\
\hline \multicolumn{5}{|l|}{ NOS score } \\
\hline$>7$ & $0.54(0.27-1.07)$ & $82.8 \%(P<0.001)$ & $0.26(0.14-0.48)$ & $28.3 \%(P=0.237)$ \\
\hline$\leq 7$ & $0.74(0.45-1.23)$ & $82.8 \%(P<0.001)$ & $0.48(0.26-0.91)$ & $87.9 \%(P<0.001)$ \\
\hline \multicolumn{5}{|c|}{ Publication year } \\
\hline$\geq 2018$ & $0.77(0.37-1.60)$ & $87.1 \%(P<0.001)$ & $0.40(0.17-0.94)$ & $88.5 \%(P<0.001)$ \\
\hline$\leq 2017$ & $0.59(0.37-0.94)$ & $78.3 \%(P<0.001)$ & $0.48(0.26-0.91)$ & $83.8 \%(P<0.001)$ \\
\hline
\end{tabular}

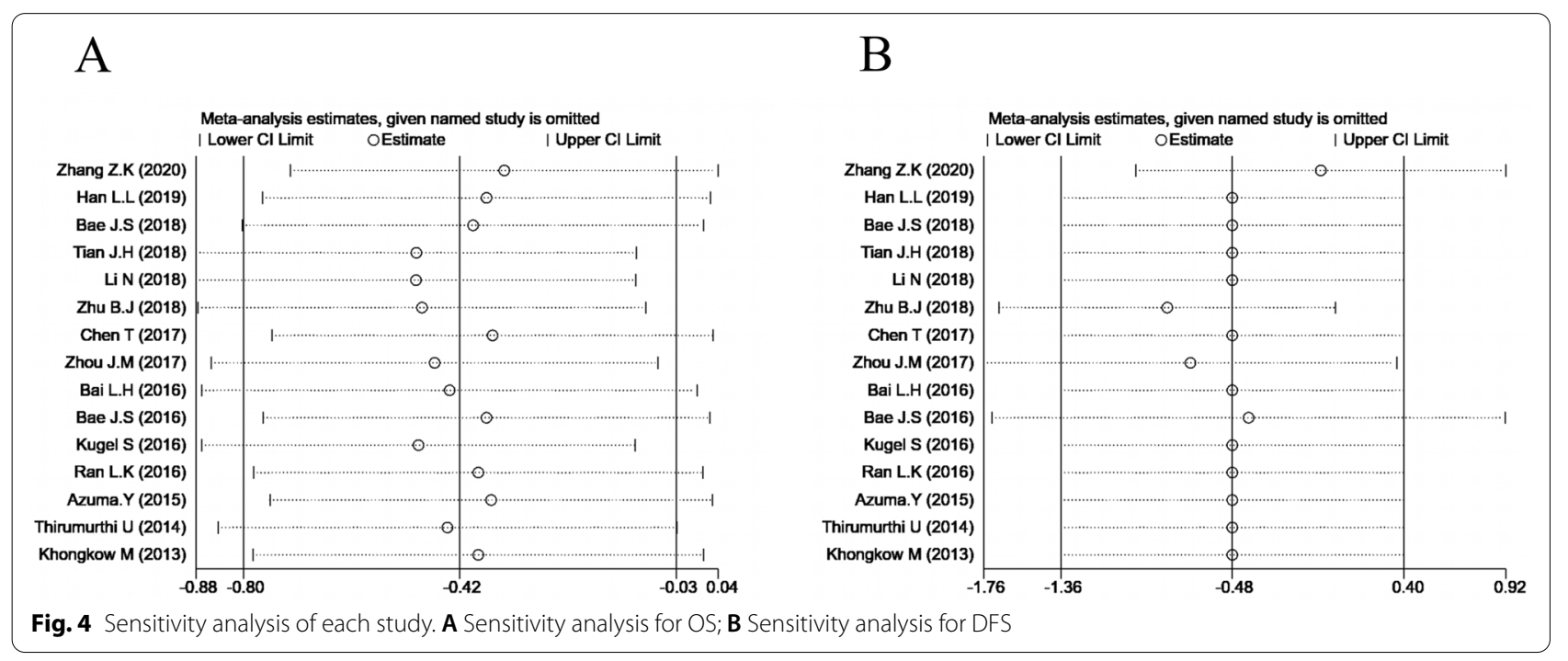


A

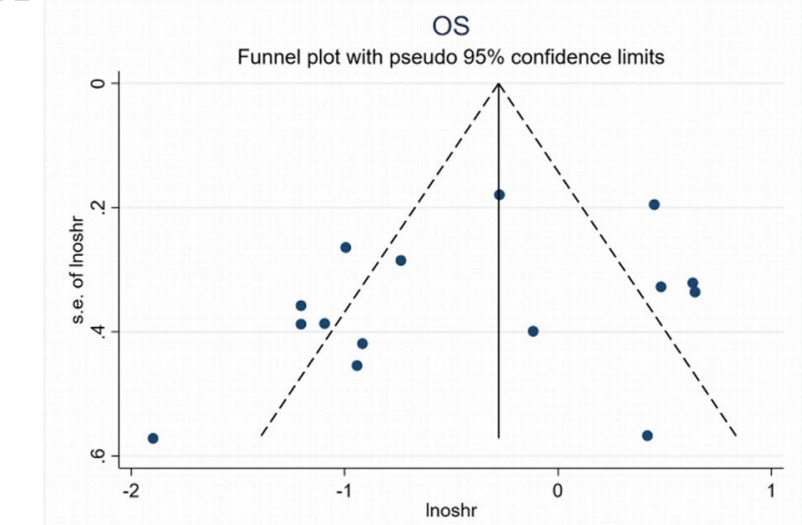

B

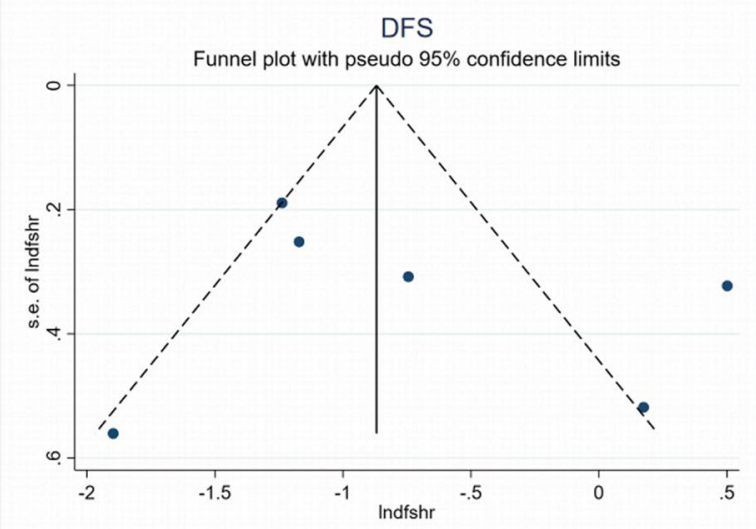

Fig. 5 Funnel plot for publication bias. A Funnel plot for OS; B Funnel plot for DFS

A

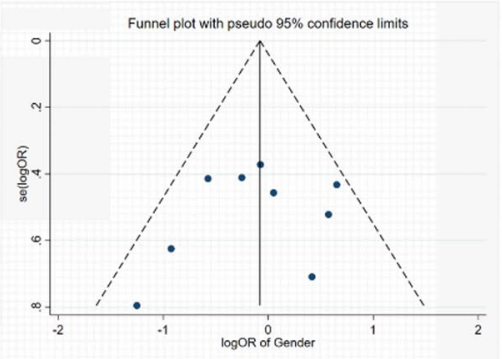

D

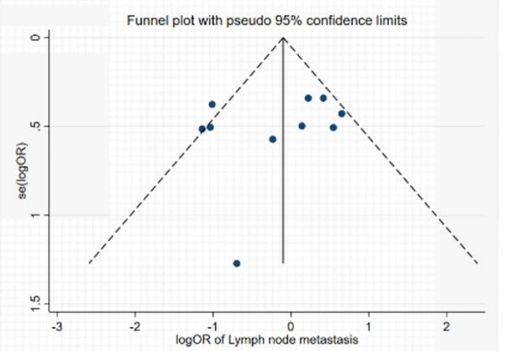

B

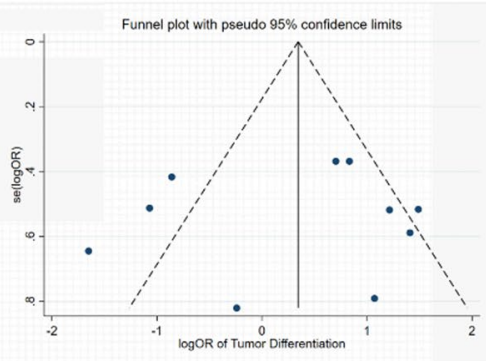

E

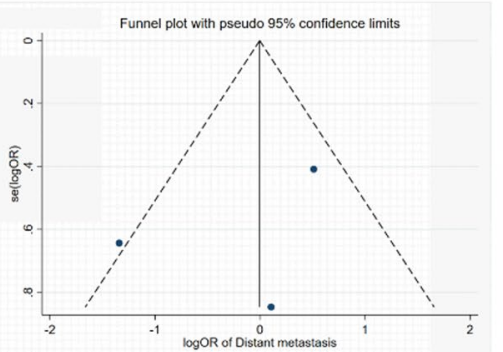

C

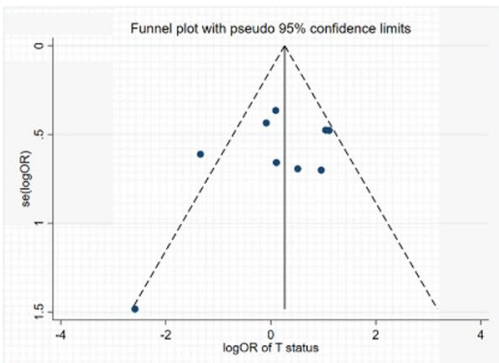

F

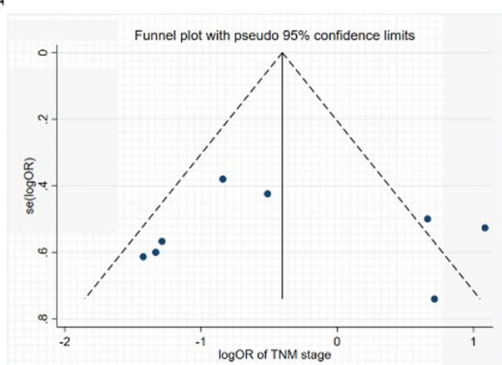

Fig. 6 Funnel plot for publication bias regarding clinicopathological features. A Gender; B Tumor differentiation; C T status; D Lymph node metastasis; E Distant metastasis; F TNM stage

gene in BLCA and UCEC using univariate Cox regression (Fig. 8).

By merging SIRT6 expression data and the survival data of all types of tumors included, we found that the low expression of SIRT6 denoted a better OS $(\mathrm{P}<0.05)$ (Fig. 9A), but was not significantly associated with worse DFS (Fig. 9B), which consistent with the meta-analysis. We also divided the data into five subgroups, including gastrointestinal cancer (including ESCA and COAD), head and neck cancer system (including HNSC and THCA), urogenital cancer (including BLCA, $\mathrm{KICH}$,
KIRC, ACC, TGCT, UCEC, CESC and USC), breast cancer and other system cancer (including GBM, THYM, UVM and $\mathrm{CHOL}$ ).

The results revealed that decreased SIRT6 was significantly associated with unfavorable OS $(\mathrm{P}<0.05)$ (Fig. 9C) and DFS $(\mathrm{P}<0.05)$ (Fig. 9D) in gastrointestinal tumors $(\mathrm{P}<0.05)$, which validated the results of metaanalysis. There was no significantly correlation between SIRT6 expression and OS (Fig. 9E) observed in breast cancer. However, downregulated SIRT6 favored better DFS in patients with breast cancer $(\mathrm{P}<0.05)$ (Fig. 9F). 


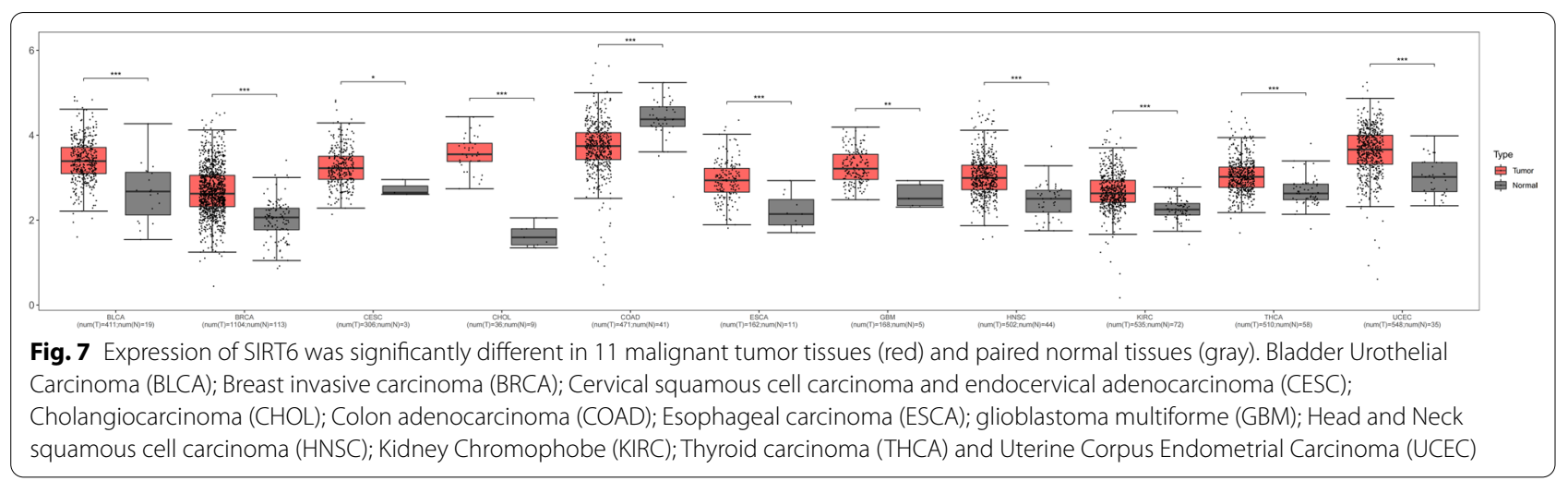

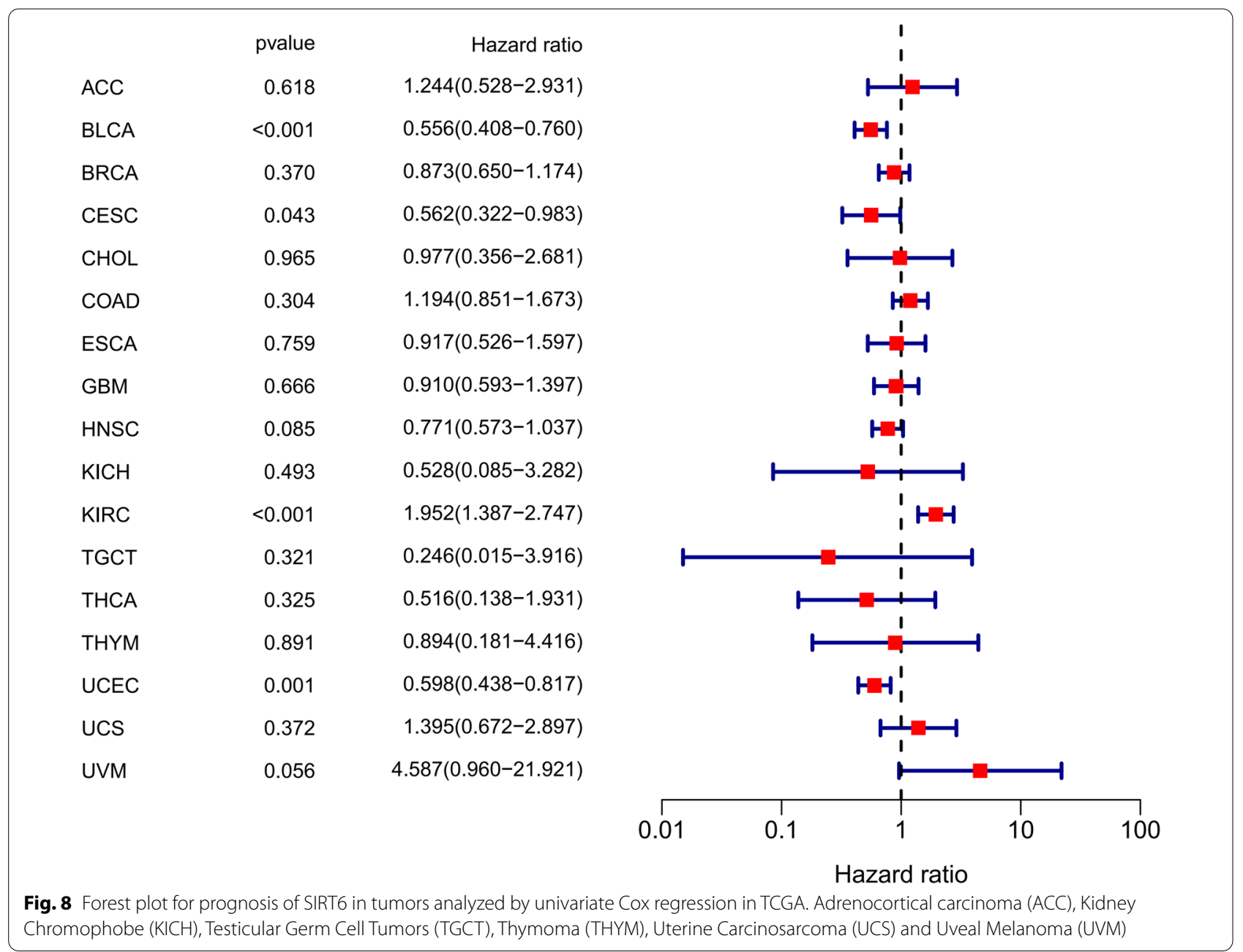

Further, low SIRT6 expression predicted worse OS in head and neck cancer $(\mathrm{P}<0.05)$ (Fig. 9G), urogenital cancer $(\mathrm{P}<0.05)$ (Fig. 9I) and other system cancer $(\mathrm{P}<0.05)$ (Fig. 9K).
We also evaluated the correlation between SIRT6 expression and clinicopathological parameters. As shown in the violin plot (Fig. 10), the expression of SIRT6 was significantly associated with clinical stage $(\mathrm{P}<0.05)$ 


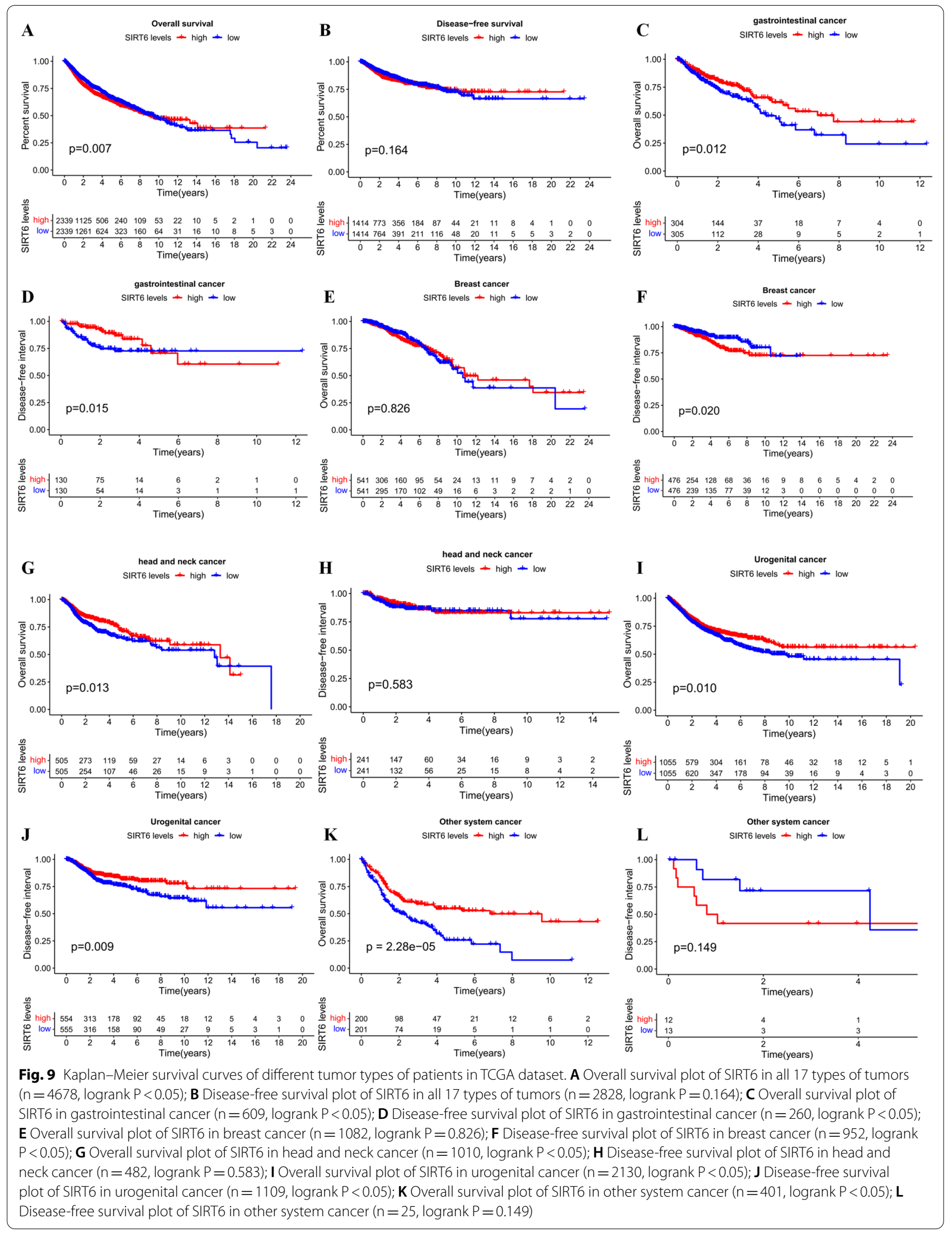



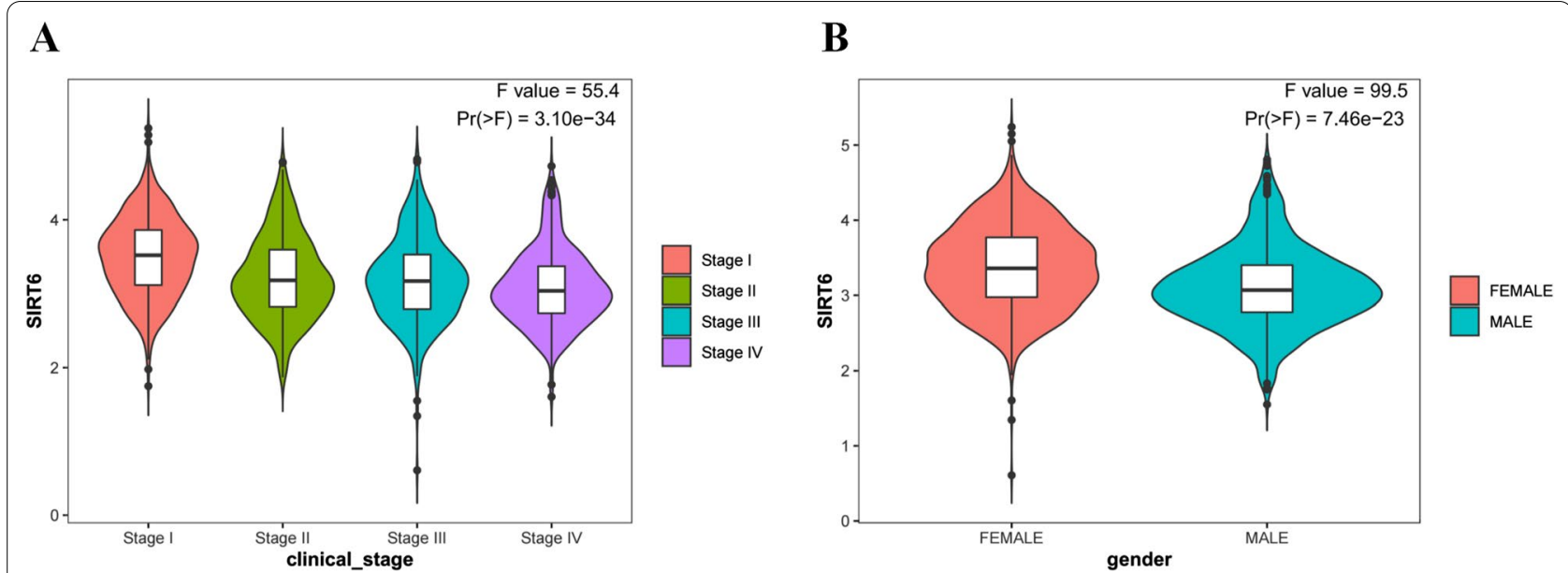

Fig. 10 Violin plot demonstrated that expression of SIRT6 was significantly associated with clinical stage $(P<0.001)$ and gender $(P<0.001)$. A Clinical stage; B Gender

and gender $(\mathrm{P}<0.05)$, but not associated with distant metastasis.

\section{Discussion}

SIRT6, a key member of the long-lived protein family, has been shown to regulate a variety of physiological processes and is intimately involved in tumour formation and progression. The current meta-analysis, which included 15 studies and 1577 patients, was the first to summarise all previously published research on the effect of SIRT6 expression on human tumour prognosis. It established a significant association between SIRT6 expression and a decline in cancer patients' OS $(\mathrm{HR}=0.66,95 \% \mathrm{CI}=0.45-$ $0.97, \mathrm{P}<0.001)$ and DFS $(\mathrm{HR}=0.48,95 \% \mathrm{CI}=0.26-0.91)$. Low SIRT6 expression was associated with a better OS in breast cancer $(\mathrm{HR}=0.49,95 \% \mathrm{CI}=0.27-0.89, \mathrm{P}=0.179)$, but was associated with a worse OS in gastrointestinal tumours $(\mathrm{HR}=0.30,95 \% \mathrm{CI}=0.10-0.91, \mathrm{P}=0.069)$.

Additionally, multivariate analysis revealed a correlation between low cytoplasmic SIRT6 expression and improved OS $(\mathrm{HR}=0.30,95 \% \mathrm{CI}=0.18-0.50, \mathrm{P}=1.000)$. SIRT6 deficiency was associated with distant metastasis $(\mathrm{OR}=2.98,95 \% \mathrm{CI}=1.59-5.57, \mathrm{P}=0.694)$. However, no obvious correlations between decreased SIRT6 expression and other clinicopathological characteristics were observed.

Additionally, the TCGA dataset was used to assess SIRT6's prognostic value in various tumour types. The TCGA dataset revealed that decreased SIRT6 was associated with a better overall survival in all 17 types of tumours, but with a worse overall survival in gastrointestinal cancer, which was consistent with meta-analysis results. However, low SIRT6 expression has been associated with a poorer OS in head and neck cancer, urogenital cancer, and other system cancers.

The biological function of SIRT6 may help to explain the contradictory findings. The current study demonstrated that SIRT6 acts as a double-edged sword during the development of solid tumours, suppressing or promoting tumour growth, depending on the type of tumour [25]. However, in the same tumor type, SIRT6 may also play dual roles in tumor progression by activating different signaling pathways, such as breast cancer $[26,27]$ and HCC [21]. In the context of cancer inhibition, the tumor suppression function of SIRT6 may achieved by regulating DNA repair, genomic stability, metabolic homeostasis and apoptosis [28], of which the main mechanism was the suppression of aerobic glycolysis (a.k.a. Warburg effect), an common alteration in glucose metabolism in cancer cells [29]. Further, upregulated SIRT6 achieves the function of tumor suppression in HCC through inhibiting phosphorylation of ERK1/2 [30] and reducing the expression of cycling D1 and p-ERK [31]. SIRT6 can not only upregulate the expression of tumor suppressors phosphatase and tensin (PTEN), and phosphatidylinositol-4,5-biphosphate (PIP2), but also can downregulate AKT1, mTOR, cyclin D1, and c-myc to inhibit the progression of colon cancer [10]. The role of SIRT6 in tumour promotion has been extensively studied in recent years. SIRT6 can inhibit Bax activation caused by H3K9 deacetylation, thereby promoting HCC growth [21]. SIRT6 promotes breast cancer by regulating the acetylation and sensitivity to lapatinib of Forkhead box protein O3 (FoxO3) [32].

Both of SIRT6's functions in the same tumour type may be related to autophagy. Autophagy can degrade toxic proteins and dysfunctional organelles in the early stages 
of cancer, but in the later stages, the sensitivity of cancer cells to pressure may be reduced, which can aid in the progression of the disease and its progression [33]. Meanwhile, SIRT6 induced DNA repair can inhibit the development of tumors in initial phase, whereas promote the growth of tumors in later phase [28].

The results of the TCGA dataset were not entirely consistent with the conclusions of the meta-analysis, which could be attributed to any of the factors listed above. Furthermore, other factors that are not mentioned in the papers, such as the detection method, the detection phase, whether or not p53 is phosphorylated, and other factors, may contribute to the differences between the results.

The original articles included in this study were all prospective, which reduced the likelihood of selection bias and reverse causation to a great extent. A large number of cases had been gathered from various studies, and the total number of participants (1577) was significant, increasing the statistical power of the analysis. The results of the funnel plot and Begg's analysis did not reveal any evidence of publication bias, indicating that the findings have a high degree of credibility. However, there were some mediocre imitations found in this study that were worth mentioning. The main imitation was the high heterogeneity between overall survival, diseasefree survival, and various clinicopathological parameter analyses. Furthermore, subgroup analysis did not yield a clear picture of the source. According to the documents and materials available, we know that while sirtuins can be found in a variety of cellular compartments, SIRT6 is primarily found in the nucleus, where it can bind and deacylate chromatin as well as other substrates, the majority of which are transcription factors (Fig. 1) [3436]. Throughout the 15 selected studies, there are only 8 articles reported the SIRT6 location, which may be a source of high heterogeneity.

Meanwhile, other possible knockoffs should be taken into consideration as well. First and foremost, we require more trials to analyze; second, we require articles that investigate a greater variety of cancer types; third, some of the survival data was extracted from Kaplan-Meier curves, which may be less reliable than a direct analysis of variance; and fourth, we require more non-English publications to search.

\section{Conclusion}

In conclusion, despite some limitations, our metaanalysis results convincingly demonstrated that decreased SIRT6 expression, as measured by immunohistochemistry, is positively associated with improved overall survival and disease-free survival in patients with solid tumours. Low SIRT6 expression may serve as a potential biomarker for improved survival outcomes in patients with a variety of solid tumours, suggesting that therapeutic approaches that directly target SIRT6 may be promising for the treatment of solid malignancies.

\section{Abbreviations \\ SIRT6: Sirtuin-6; OS: Overall survival; DFS: Disease-free survival; RFS: Recur- rence-free survival; TCGA: The Cancer Genome Atlas; PTC: Papillary thyroid can- cer; RCC: Renal cell carcinoma; Cl: Confidence interval; $1^{2}$ : Inconsistency index; HR: Hazard ratios; NOS: Newcastle Ottawa Scale; IHC: Immunohistochemistry; OC: Ovarian carcinomas; CRC: Colon cancer; NSCLC: Non-small cell lung cancer; GC: Gastric cancer; PDAC: Pancreatic ductal adenocarcinoma; HCC: Hepatocellular carcinoma; BRC: Breast cancer; OS: Osteosarcoma; Nu: Nucleus; Cy: Cytoplasm.}

\section{Supplementary Information}

The online version contains supplementary material available at https://doi. org/10.1186/s12935-022-02511-3.

Additional file 1: Figure S1. Forest plot for different clinicopathological parameters.

Additional file 2: Figure S2. Forest plot for OS of different clinicopathological parameters.

Additional file 3: Figure S3. Forest plot for DFS of different clinicopathological parameters.

Additional file 4: Table S1. Summarized data of clinical and pathological parameters from the eligible studies.

\section{Acknowledgements}

Not applicable.

Authors' contributions

XJW, SYW: protocol, project development, formal analysis, writing-original draft; YXZ, KMN, SZL: data collection, data analysis, writing —-reviewing and editing; $Z Y, X Z Z$ : quality assessment; $Z C L, ~ L N L$ : methodology, software; $R X$, $X Y Z, X Y$ : data curation; $X Y L, X P Z$ : resources, supervision; $W C$ : resources, supervision, project administration; CZZ: conceptualization, resources, supervision, funding acquisition, writing-original draft. All authors read and approved the final manuscript.

\section{Funding}

This research was funded by the Key R\&D Projects in the Tianjin Science and Technology Pillar Program Grant (number 19YFZCSY00420), National key R\&D Program of China Grant (number 2017YFC1700604), National key R\&D Program of China Grant (number 2017YFC1700606) and Tianjin Key Medical Discipline (Specialty) Construction Project.

\section{Availability of data and materials}

The datasets of meta-analysis used in this study are available from the corresponding author upon reasonable request. The data in TCGA analysis are available at https://www.cancer.gov/about-nci/organization/ccg/research/ structural-genomics/tcga and https://xena.ucsc.edu/.

\section{Declarations}

Ethics approval and consent to participate

Not applicable.

Consent for publication

Not applicable. 


\section{Competing interests}

The authors declared that no competing of interests existing in this study.

\section{Author details}

${ }^{1}$ Department of Colorectal Surgery, Tianjin Union Medical Center, Tianjin 300121, China. ${ }^{2}$ School of Medicine, Nankai University, Tianjin 300071 , China. ${ }^{3}$ School of Integrative Medicine, Tianjin University of Traditional Chinese Medicine, Tianjin 301617, China. ${ }^{4}$ School of Mathematical Sciences and LPMC, Nankai University, Tianjin 300071, China. ${ }^{5}$ Tianjin Institute of Coloproctology, Tianjin 300121, China. ${ }^{6}$ Tianjin Medical University, Tianjin 300041, China.

Received: 3 October 2021 Accepted: 3 February 2022

Published online: 16 February 2022

\section{References}

1. Sosnowska B, Mazidi M, Penson P, Gluba-Brzozka A, Rysz J, Banach M. The sirtuin family members SIRT1, SIRT3 and SIRT6: their role in vascular biology and atherogenesis. Atherosclerosis. 2017;265:275-82.

2. Lerrer B, Gertler AA, Cohen HY. The complex role of SIRT6 in carcinogenesis. Carcinogenesis. 2016:37(2):108-18.

3. Dong Z, Lei Q, Liu L, Cui H. Function of SIRT6 in tumor initiation and progression. Sheng Wu Gong Cheng Xue Bao. 2016:32(7):870-9.

4. Chen T, Sun Z, Liu F, Wang Q. RASSF1A and SIRT6 in non-small cell lung cancer: relationship with clinical outcome. Oncol Lett. 2017;14(5):5759-64

5. Lin H, Hao Y, Zhao Z, Tong Y. Sirtuin 6 contributes to migration and invasion of osteosarcoma cells via the ERK1/2/MMP9 pathway. FEBS Open Bio. 2017;7(9):1291-301

6. Qu N, Hu JQ, Liu L, Zhang TT, Sun GH, Shi RL, et al. SIRT6 is upregulated and associated with cancer aggressiveness in papillary thyroid cancer via BRAF/ERK/Mcl1 pathway. Int J Oncol. 2017;50(5):1683-92.

7. Liu Y, Xie QR, Wang B, Shao J, Zhang T, Liu T, et al. Inhibition of SIRT6 in prostate cancer reduces cell viability and increases sensitivity to chemotherapeutics. Protein Cell. 2013;4(9):702-10.

8. Jeh SU, Park JJ, Lee JS, Kim DC, Do J, Lee SW, et al. Differential expression of the sirtuin family in renal cell carcinoma: aspects of carcinogenesis and prognostic significance. Urol Oncol. 2017;35(12):675.

9. Kugel S, Sebastian C, Fitamant J, Ross KN, Saha SK, Jain E, et al. SIRT6 suppresses pancreatic cancer through control of Lin28b. Cell. 2016;165(6):1401-15.

10. Tian J, Yuan L. Sirtuin 6 inhibits colon cancer progression by modulating PTEN/AKT signaling. Biomed Pharmacother. 2018;106:109-16.

11. Bai L, Lin G, Sun L, Liu Y, Huang X, Cao C, et al. Upregulation of SIRT6 predicts poor prognosis and promotes metastasis of non-small cell lung cancer via the ERK1/2/MMP9 pathway. Oncotarget. 2016;7(26):40377-86.

12. Shen $X, L i P, X u Y$, Chen $X$, Sun $H$, Zhao $Y$, et al. Association of sirtuins with clinicopathological parameters and overall survival in gastric cancer. Oncotarget. 2017:8(43):74359-70.

13. Tierney JF, Stewart LA, Ghersi D, Burdett S, Sydes MR. Practical methods for incorporating summary time-to-event data into meta-analysis. Trials. 2007:8:16.

14. Zhang Z, Ha SH, Moon YJ, Hussein UK, Song Y, Kim KM, et al. Inhibition of SIRT6 potentiates the anti-tumor effect of doxorubicin through suppression of the DNA damage repair pathway in osteosarcoma. J Exp Clin Cancer Res. 2020;39(1):247.

15. Han LL, Jia L, Wu F, Huang C. Sirtuin6 (SIRT6) promotes the EMT of hepatocellular carcinoma by stimulating autophagic degradation of E-cadherin. Mol Cancer Res. 2019;17(11):2267-80.

16. Bae JS, Noh SJ, Kim KM, Park SH, Hussein UK, Park HS, et al. SIRT6 is involved in the progression of ovarian carcinomas via beta-cateninmediated epithelial to mesenchymal transition. Front Oncol. 2018;8:538

17. Li N, Mao D, Cao YS, Li H, Ren F, Li KY. Downregulation of SIRT6 by miR$34 c-5 p$ is associated with poor prognosis and promotes colon cancer proliferation through inhibiting apoptosis via the JAK2/STAT3 signaling pathway. Int J Oncol. 2018;52(5):1515-27.

18. Zhu BJ, Yan YJ, Shao BY, Tian LW, Zhou WH. Downregulation of SIRT6 is associated with poor prognosis in patients with non-small cell lung cancer. J Int Med Res. 2018:46(4):1517-27.
19. Zhou JM, Wu A, Yu XT, Zhu JW, Dai H. SIRT6 inhibits growth of gastric cancer by inhibiting JAK2/STAT3 pathway. Oncol Rep. 2017;38(2):1059-66.

20. Bae JS, Park SH, Jamiyandorj U, Kim KM, Noh SJ, Kim JR, et al. CK2alpha/ CSNK2A1 phosphorylates SIRT6 and is involved in the progression of breast carcinoma and predicts shorter survival of diagnosed patients. Am J Pathol. 2016;186(12):3297-315

21. Ran LK, Chen Y, Zhang ZZ, Tao NN, Ren JH, Zhou L, et al. SIRT6 overexpression potentiates apoptosis evasion in hepatocellular carcinoma via BCL2-associated X protein-dependent apoptotic pathway. Clin Cancer Res. 2016;22(13):3372-82

22. Azuma Y, Yokobori T, Mogi A, Altan B, Yajima T, Kosaka T, et al. SIRT6 expression is associated with poor prognosis and chemosensitivity in patients with non-small cell lung cancer. J Surg Oncol. 2015;112(2):231-7.

23. Thirumurthi U, Shen J, Xia W, LaBaff AM, Wei Y, Li CW, et al. MDM2mediated degradation of SIRT6 phosphorylated by AKT1 promotes tumorigenesis and trastuzumab resistance in breast cancer. Sci Signaling. 2014;7(336):ra71.

24. Khongkow M, Olmos Y, Gong C, Gomes AR, Monteiro LJ, Yague E, et al. SIRT6 modulates paclitaxel and epirubicin resistance and survival in breast cancer. Carcinogenesis. 2013;34(7):1476-86.

25. Fiorentino F, Carafa V, Favale G, Altucci L, Mai A, Rotili D. The two-faced role of SIRT6 in cancer. Cancers (Basel). 2021;13(5):1156.

26. Choe M, Brusgard JL, Chumsri S, Bhandary L, Zhao XF, Lu S, et al. The RUNX2 transcription factor negatively regulates SIRT6 expression to alter glucose metabolism in breast cancer cells. J Cell Biochem. 2015;116(10):2210-26.

27. Khongkow M, Olmos Y, Gong C, Gomes AR, Monteiro LJ, Yagüe E, et al. SIRT6 modulates paclitaxel and epirubicin resistance and survival in breast cancer. Carcinogenesis. 2013;34(7):1476-86.

28. Liu G, Chen H, Liu H, Zhang W, Zhou J. Emerging roles of SIRT6 in human diseases and its modulators. Med Res Rev. 2021:41(2):1089-137.

29. Sebastián C, Zwaans BM, Silberman DM, Gymrek M, Goren A, Zhong L, et al. The histone deacetylase SIRT6 is a tumor suppressor that controls cancer metabolism. Cell. 2012;151(6):1185-99.

30. Zhang ZG, Qin CY. Sirt6 suppresses hepatocellular carcinoma cell growth via inhibiting the extracellular signal-regulated kinase signaling pathway. Mol Med Rep. 2014;9(3):882-8.

31. Wang Y, Pan T, Wang H, Li L, Li J, Zhang D, et al. Overexpression of SIRT6 attenuates the tumorigenicity of hepatocellular carcinoma cells. Oncotarget. 2017:8(44):76223-30

32. Mahmud Z, Gomes AR, Lee HJ, Aimjongjun S, Jiramongkol Y, Yao S, et al. EP300 and SIRT1/6 Co-regulate lapatinib sensitivity via modulating FOXO3-acetylation and activity in breast cancer. Cancers (Basel). 2019;11(8):1067.

33. Garcia-Peterson LM, Guzmán-Pérez G, Krier CR, Ahmad N. The sirtuin 6: an overture in skin cancer. Exp Dermatol. 2020;29(2):124-35.

34. Michishita E, Park JY, Burneskis JM, Barrett JC, Horikawa I. Evolutionarily conserved and nonconserved cellular localizations and functions of human SIRT proteins. Mol Biol Cell. 2005;16(10):4623-35.

35. Tennen RI, Berber E, Chua KF. Functional dissection of SIRT6: identification of domains that regulate histone deacetylase activity and chromatin localization. Mech Ageing Dev. 2010;131(3):185-92.

36. Kawahara TL, Rapicavoli NA, Wu AR, Qu K, Quake SR, Chang HY. Dynamic chromatin localization of Sirt6 shapes stress- and aging-related transcriptional networks. PLoS Genet. 2011;7(6):e1002153.

\section{Publisher's Note}

Springer Nature remains neutral with regard to jurisdictional claims in published maps and institutional affiliations. 